\title{
A Planning Framework for Optimal Partitioning of Distribution Networks into Microgrids
}

\begin{abstract}
This paper proposes a novel methodology for the optimal design of microgrids in distribution systems with multiple distributed generation units. Following the IEEE Standard 1547.42011, the operation and control of large distribution networks can be enhanced by dividing these networks into multiple virtual microgrids. The proposed planning framework incorporates the necessary conditions for microgrids to operate efficiently in gridconnected operating mode and successfully during islanding. To obtain a robust design, the clustering process considers three objectives: maximizing the self-adequacy of the designed microgrids, maximizing microgrid islanding success probability, and a combination of both targets. For this purpose, the PG\&E distribution system with 69 buses is selected as a test case. Backtracking search optimization algorithm, a probabilistic load flow approach, and graph-based theories are used to accomplish this research. Simulation results demonstrate the effectiveness of combining the self-adequacy and the islanding success probability objectives in the clustering process. Compared with other strategies present in the previous literature, the proposed framework results in more self-sufficient and successful islands assessed in terms of active and reactive power adequacy as well as voltage constraints. Next, the effects of increased penetration level of distributed generation units and installation of both distributed energy storage units and distributed reactive sources on the design process are examined. Finally, comparison with other microgrid design objectives applied in previous researches reveals that the resultant design is sensitive to the system's reliability, security, and economic requirements.
\end{abstract}

Index Terms-Backtracking search, clustering, islanding, microgrid design, optimization, reactive sources, self-adequacy, storage units.

\section{INTRODUCTION}

$\mathrm{T}$ HE recent significant advancement in technology has led to considerable efforts to transform conventional electric power grids into modern small-scale networks where consumers become prosumers, so-called microgrids [1]. A simple definition of a microgrid is a group of connected distributed resources and loads. Microgrids are mainly fueled by renewables and managed by smart energy management systems [2]. Microgrids offer a solution to overcome numerous challenges associated with integrating more renewable resources in conventional electrical systems [3] - [5]. Employment of microgrids is expected to increase the reliability, efficiency, and flexibility of distribution systems in a cost-effective manner. The design, control, and operation of microgrids are still challenging, and hence extensive research is focused on tackling these issues [6] - [9]. The ability of a microgrid to quickly isolate and operate independently, in case of any disturbance in the larger grid to which the microgrid is connected, is one of the most distinctive features of a microgrid [10].

The IEEE standard (std. 1547), which was compiled in 2003, introduced the microgrid as the main component of active distribution networks [11]. Following the release of this standard, several research papers were conducted on the partitioning of distribution networks into islands particularly in the event of a fault or a disturbance. In [12], the authors programmed a multi-stage stochastic model to achieve minimum loss of load. Effects of island partitioning on a distribution system with multi-point faults were investigated. In [13], an island partitioning strategy based on energy risk evaluation using supply-demand balance was proposed. For a distribution system with a high penetration level of distributed generation units $(D G s)$, the reliability of power supply was considered in [14]. An island partitioning strategy that reduces the effect of node voltage fluctuation on stability during power restoration was also introduced. Restoration of important loads was set as the primary goal through the identification of vulnerable nodes. In 2011, the modified IEEE standard (std. 1547.4) was released [15]. After that, the concept of partitioning of distribution systems into networked microgrids has been extended to planning studies through the optimal microgrid design. In [16], the concept of coupled microgrids was expanded for the realization of smart grid functions such as improved reliability, active control of the load, improved generation efficiency, and self-healing actions. Using the CERTS/AEP microgrid test bed, it was demonstrated that the best way to control distribution systems with enormous numbers of intermittent resources and loads is to partition these systems into clusters optimally. The complexity of rapid control, during normal operation and in case of any disturbance, could be reduced by the use of coupled microgrids. A salient advantage is that microgrids could be re-dispatched using only information on the power available for dispatch regardless of the details on each resource. The response to a control request is made internally within the microgrid either by increasing its internal generation or shedding loads of the least importance. Ref. [17] - [20] studied various uncertainties that the microgrid planning problem is subjected to, which obstruct the outspread of this viable technology such as uncertainties in renewable 
generation, load demand, and energy prices. In [21], a stochastic AC/DC microgrid planning model was illustrated. The optimal capacity of distributed energy resources, as well as the optimal generation mix, were determined based on economic considerations. A stochastic risk-based bilevel model for optimal planning of distribution networks was proposed in [22]. The upper level of this model transferred a conventional distribution system into an active system through the optimal placement of distributed energy resources. The lower level applied the cuckoo optimization algorithm and imperialist competitive algorithm for optimal switch allocation to partition a distribution system into interconnected microgrids. In [23], minimization of the energy, not supplied in both grid-connected and islanded modes of microgrids, was taken into consideration. The authors in [24] formulated two different optimization problems to study the optimal partitioning of the IEEE 33-bus distribution system into supply-sufficient microgrids. The first problem involved increasing the penetration level of $D G s$ for the system under study to improve its performance in terms of energy losses and voltage profile. The second problem considered partitioning of the test system to minimize the generation-load imbalance within microgrids as well as the power exchange between microgrids. The problem was solved using mixed-integer linear programming considering only the presence of sufficient active power generation for the success of microgrids. Ref. [25] investigated the optimal placement of energy storage units (ESUs) and reactive sources (RSs) with the construction of microgrids. Construction of microgrids in [25] was solved using tabu search (TS) taking into account the power imbalance within the microgrids as a single objective. The aforementioned problem was solved in [26] using TS considering reliability indices. The design in [27] scrutinized the communication and control requirements for partitioning of distribution networks using $T S$. In [28], the operation of future distribution networks based on multi-microgrids approach was augmented based on several technical criteria including active power adequacy, active power losses, reliability, and voltage profile. The reactive power adequacy has not been taken into consideration.

In this paper, the optimal partitioning of active distribution networks into interconnected microgrids has been revisited. A new objective is proposed which takes into consideration the necessary conditions for each microgrid to operate with minimum interaction with other microgrids in grid-connected operation and successfully if islanding is required. These conditions have not been addressed simultaneously in the existing literature. The recently developed backtracking search optimization (BTSO) is chosen as the solution algorithm [29]. The validity of the BTSO in solving the partitioning of distribution networks problem is demonstrated by comparing to the $T S$, as recently reported in [24].

The significance of this work can be highlighted as follows: applying a novel objective function for the optimal microgrid design problem that maximize the self-adequacy of the designed microgrids and maximizes the probability of successful islanding operation if required; the newly proposed objective function has not been addressed in the previous literature; verifying the validity of the $B T S O$ as a solution algorithm; comparing the results obtained by BTSO to results obtained by $T S$ that were recently reported in previous researches; taking into account the stochastic nature of renewables and load demand through probabilistic modeling of system components; examining the sensitivity of the design to several factors such as: increased penetration level of $D G s$, installation of ESUs and RSs, technical criteria considered during the design such as reliability, adequacy, supply security and others that can significantly affect the operation of active distribution networks.

\section{PARTITIONING OF DISTRIBUTION SYSTEMS CONCEPT}

In this paper, a methodological approach is presented for partitioning of distribution systems. Incentives and benefits of the chosen objectives are revealed in the following subsections.

\section{A. Incentives for the Proposed Design}

Despite promising benefits of grid-connected renewable $D G s$, utilities are facing new challenges to the security, reliability, power quality, and protection of power networks. A microgrid, as a new emerging technology, has been considered as one of the ideal solutions to these problems. A microgrid is a small power network comprising energy resources and loads. A microgrid can be supplied from a medium-voltage or a lowvoltage grid and can also supply the grid with surplus power. In emergencies such as faults, a microgrid can isolate itself from the rest of the network and supply its local loads, i.e., islanding operation. These important features of microgrids can make modern distribution networks more reliable and more secure and can enhance the quality of power for utilities and customers. Fig. 1 explains the concept of clustering of a distribution network into a number of microgrids.

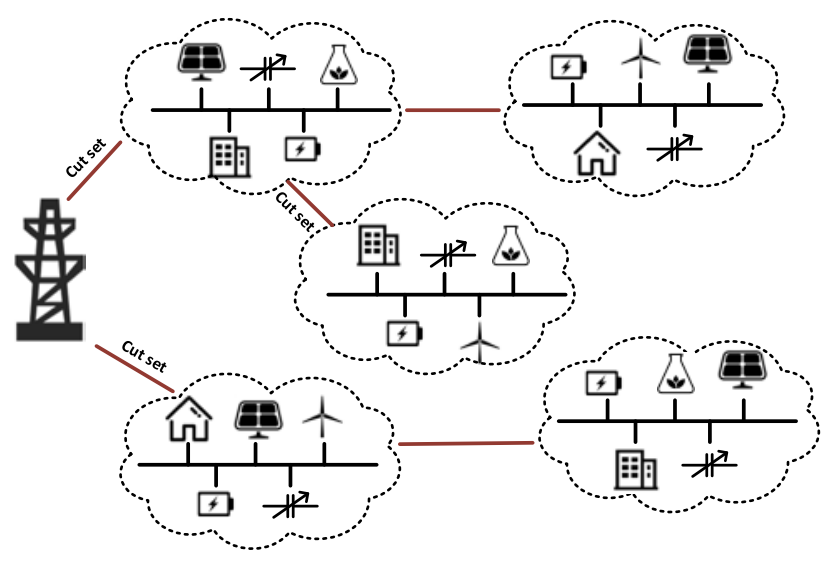

Fig. 1. Clustering of a distribution network into microgrids.

Transforming costly centralized networks into multiple islands can provide enhanced operation and control [30]. The questions that this paper aims to answer are: how to optimally partition traditional distribution networks, what factors should be taken into consideration during the microgrid design process, and how sensitive is the optimal design to future network upgrades. 


\section{B. Self-Adequacy of Microgrids}

One of the essential factors to be considered in the design process is maximizing the self-adequacy of microgrids. During grid-connected operation, the designed microgrids should operate with minimum interaction with each other. Minimizing the energy exchange between the microgrids can be achieved by optimizing the transferred energy on the interconnecting power lines. In the case of emergencies, splitting distribution networks into self-sufficient microgrids can prevent the propagation of a disturbance and thus avoid an unforeseen chain of events. Also, a minimum number of self-healing actions will be then required [31]. Maximizing self-adequacy during design will lead to maximizing the microgrids' self-sufficiency and supply security during operation.

\section{Successful Islanding Operation of Microgrids}

Unintentional islanding can happen due to unexpected faults or other unscheduled events which may lead to the loss of the main grid supply. In this case, a microgrid is required to serve its critical loads during islanding. The successful operation of islanded microgrids is a crucial factor for utility planners to improve the reliability of distribution systems. The uncertainty of renewables in a microgrid has a significant impact on the success of these islands. Thus, during the design process, it is crucial to take into consideration the rate at which a microgrid can operate successfully in the case of islanding.

\section{MODELING OF SYSTEM COMPONENTS}

In this section, a typical probabilistic model is adopted that accounts for the uncertainties of generated power from renewables such as wind turbines (WTs) and photovoltaics $(P V s)$ as well as the uncertainties in the load profile [32] - [34]. Probability density functions ( $P D F s$ ) available in the previous literature are used to represent the stochastic variables. Rayleigh $P D F$ and the beta $P D F$ are used for modeling of the wind speed [35] and the solar irradiance [36] respectively. Load demand follows the IEEE-RTS model in [37]. In this paper, an assumption is made that all biomass $(B M)$ units are considered as a dispatchable generation with constant power. However, the output power of $B M$ units can be modeled in the same way as $W T s$ and $P V S$ using a suitable PDF that represents the availability of $B M$ during the planning period. The $R S S$ are modeled as reactive sources supplying constant reactive power. The ESUS are represented as generators during on-peak hours, i.e., discharging period, and as loads during off-peak hours, i.e., charging period [45].

The modeling process starts with the collection of data for the studied period over the four seasons, i.e., one year in this paper. Next, $P D F s$ for probabilistic variables are generated for each season [38]. The PDFs are then divided into states. Every state has its probability $\left(\delta_{\text {state }}\right)$. The value of $\delta_{\text {state }}$ can be evaluated by estimating the area under the graph. The higher the number of states, the more accurate the solution will be. However, the simulation can be more complex and a longer computational time will be required. The probabilistic output power of WTs and PVs is calculated for each state using $\delta_{\text {state }}$.
Probabilistic power flows in the lines are obtained by running the steady state load flow that corresponds to each state individually and accumulating the power flows using $\delta_{\text {state }}$.

\section{OBJECTIVE FUNCTIONS AND CONSTRAINTS}

Partitioning of a distribution network involves determining the virtual cut-set lines that minimize or maximize the desired objective [38]. In this paper, we consider three objective functions for optimization. These objective functions, as well as the associated constraints, are elaborated in this section.

\section{A. First Objective Function}

This objective considers minimizing the active $(P)$ and reactive $(Q)$ power flows in the lines connecting the microgrids, which is a step towards self-sufficient islands. As mentioned earlier, this objective aims hto minimize the interaction of the microgrids with each other during the grid-connected mode of operation as follows;

$\operatorname{Min} F_{1}=K_{1} \times P_{\text {norm }}+K_{2} \times Q_{\text {norm }}$

$0 \leq K_{1}, K_{2} \leq 1, K_{1}+K_{2}=1$

where $P_{\text {norm }}$ and $Q_{\text {norm }}$ are the normalized probabilistic power indices of the microgrids' interconnecting lines. The indices can be evaluated by applying the probabilistic load flow algorithm for the system under study. The parameters $K_{1}$ and $K_{2}$ can be selected depending on the microgrid requirements with regard to balancing the $P$ and $Q$ powers.

$P_{\text {norm }}=\sum_{i=1}^{N_{\text {states }}}\left|P_{\text {cutset }_{i}}\right| \times \delta_{i} /\left(N_{\text {microgrids }}-1\right)$
$Q_{\text {norm }}=\sum_{i=1}^{N_{\text {states }}}\left|Q_{\text {cutset }_{i}}\right| \times \delta_{i} /\left(N_{\text {microgrids }}-1\right)$

where $P_{\text {cutset }}$ and $Q_{\text {cutset }}$ are the active and reactive power flows in the cut-set line joining two microgrids in the state $i$, $N_{\text {states }}$ represents the total number of states in the selected period, i.e., one year, $\delta_{i}$ is the probability of state $i, N_{\text {microgrids }}$ indicates the number of microgrids into which the system will be split, and the power flows are normalized by the term $\left(N_{\text {microgrids }}-1\right)$.

\section{B. Second Objective Function}

The ability of a microgrid to operate successfully during islanding operation depends mainly on having enough $P$ and $Q$ resources to supply its critical loads and to preserve the frequency and the voltage of each bus within acceptable deviation limits [44]. Another essential requirement in [39] is that more than $60 \%$ of a microgrid's generated power must be supplied from dispatchable $D G s$, i.e., $B M$ in this paper.

$P_{G} \geq P_{C}, Q_{G} \geq Q_{C}, P_{b i o} \geq 0.6 \times P_{D G s}$

where $P_{G}$ and $P_{C}$ are the generated and consumed active power in a microgrid respectively, $Q_{G}$ and $Q_{c}$ are the generated and consumed reactive power in a microgrid respectively, $P_{b i o}$ is the active power generated by all dispatchable $B M$ units, and $P_{D G S}$ is the active power produced by all $D G s$ in a 
microgrid. These necessary conditions presented in (3) are achieved during the design process by the gradual shedding of loads until all conditions are satisfied. The reliability requirements of loads must be taken into consideration, i.e., shedding of smaller loads first. A new indicator for the islanding success of each microgrid $I N D_{M G i}$ is proposed.

$I N D_{M G i}=\left\{\begin{array}{ccc}1 & P_{G_{i}} \geq P_{c_{i}} & Q_{G_{i}} \geq Q_{c_{i}} \\ & V_{\text {min }_{i}} \leq V_{i} \leq V_{\text {max }_{i}} & P_{\text {bio }_{i}} \geq 0.6 \times P_{D G s_{i}} \\ 0 & \text { otherwise } & \end{array}\right.$

where $I N D_{M G i}$ is the microgrid's success indicator during the $i^{\text {th }}$ state, and $V_{i}$ is the voltage of all buses in the microgrid in the $i^{t h}$ state. The indicator for an individual microgrid can be calculated for each state $\left\{1,2,3 \ldots, \mathrm{i}, \ldots, N_{\text {states }}\right\}$ and accumulated as follows;

$I N D_{M G}=\left(\sum_{i=1}^{N_{\text {states }}} I N D_{M G_{i}} \times \delta_{i}\right) /\left(\sum_{i=1}^{N_{\text {states }}} \delta_{i}\right)$

For a system with many microgrids, the objective function to be maximized $F_{2}$ is $I N D_{\text {sys }}$, which is the weighted sum of $I N D_{M G}$ of all microgrids.

$\operatorname{Max} F_{2}=I N D_{s y s}=\frac{\left(\sum_{j=1}^{\text {Nmicrogrids }}{ }_{I N D_{M G}} \times \text { NoL }_{j}\right)}{\left(\sum_{j=1}^{\text {Nmicrogrids }} \operatorname{NoL}_{j}\right)}$

where $N o L_{j}$ represents the number of load points in a microgrid.

\section{Combining objectives}

A novel objective function $F_{3}$ is introduced which combines the first objective, i.e., self-adequacy and the second objective, i.e., islanding success probability as a weighted summation.

$\operatorname{Min} F_{3}=a \times F_{1}+b \times\left(1-F_{2}\right)$

$0 \leq a, b \leq 1, a+b=1$

As shown in (7), the objective function $F_{3}$ to be minimized is the weighted summation of $F_{1}$ and $\left(1-F_{2}\right)$. The values of the weights $a$ and $b$ determine whether $F_{3}$ targets the microgrid adequacy, microgrid islanding success, or a combination of both.

\section{Optimization Constraints}

For all objectives, a set of system-topology-related constraints has to be satisfied after partitioning such as all the designed microgrids have a radial configuration, there are no isolated buses, and that all buses are included in all microgrids. The shortest path algorithm [40] is adopted to ensure that these conditions are satisfied. Other constraints should be satisfied during the design process as follows:

\section{1) Voltage limits}

The voltage magnitudes of all buses in a microgrid should be kept within pre-specified limits.

$\left|V_{\min }\right| \leq\left|V_{k-i}\right| \leq\left|V_{\max }\right| \quad, \mathrm{k} \neq 1$ where $V_{k-i}$ is the voltage magnitude of bus $k$ in any state $i, V_{\min }$ and $V_{\text {max }}$ are the minimum and maximum magnitudes of bus voltages for all buses except the root bus, i.e., $\left|V_{1}\right|=1$.

\section{2) Line capacity limit}

The power flow of each line in a microgrid is limited to the maximum thermal capacity.

$\left|I_{\text {line }}\right| \leq\left|I_{\text {max }}\right|$

where $I_{\max }$ is the maximum allowable line current so that no overloading occurs.

\section{3) Power generated by ESUs}

For each microgrid, the total active power generated by all storage units at any state must be less than total active load demand.

$P_{E S U S-j, i} \leq P_{\text {load }-j, i}$

where $P_{E S U S-j, i}$ is the total active power generated by all ESUs and $P_{\text {load-j,i }}$ is the total active power consumed in the microgrid $j$ in the state $i$.

The work in this paper is related to the planning stage in steady-state conditions. The operating strategies, transients, dynamics, and stability problems associated with the transition of microgrids from grid connected to islanding mode of operation [43] are outside the scope of this paper.

\section{BACKTRACKING SEARCH OPTIMIZATION}

As a result of the large search space, identifying the optimal electrical boundaries of microgrids is a complex problem. Heuristic algorithms are the most suitable for this level of complexity. This paper applies the BTSO, which is a recently developed iterative optimization technique [29]. The fundamental steps of the population-based BTSO are highlighted in Fig. 2.

The $B T S O$ algorithm starts with the initiation of a population matrix $\mathbf{P}$ for the control variables of the studied problem. The control variables in this paper are the virtual cut-set lines that define the boundaries of the designed microgrids.

$$
\begin{array}{r}
\mathbf{P}_{\mathbf{i} . \mathbf{j}} \leftarrow U\left(\min _{j}, \max _{j}\right) \quad i=\{1,2,3, \ldots N p o p\} \\
j=\{1,2,3, \ldots \text { Dim }\}
\end{array}
$$

where $U$ is the rectangular distribution, $\min , \max$ are the minimum and maximum limits of the virtual cut-set lines, and Npop ,Dim are the population size and problem dimension respectively.

A historical population matrix of cut-set lines oldP, is then generated randomly. At the start of every iteration, the oldP matrix is permuted randomly. The permutation step shuffles the order of control variables in the historical population matrix.

$\operatorname{oldP}_{\mathbf{i}, \mathbf{j}} \leftarrow U\left(\right.$ low $\left._{j} . u p_{j}\right)$

oldP $\leftarrow$ permute $($ oldP) 
Using mutation and crossover, a new population of solutions $\mathbf{T}$ is generated for every iteration based on the experience from the preceding generations. The matrix oldP is used to evaluate the search-directing matrix (oldP $-\mathbf{P})$.

$$
\mathbf{T}=(F \times \operatorname{map} \times(\text { oldP }-\mathbf{P}))+\mathbf{P}
$$

where $F$ controls the amplitude of the search-directing matrix, and map is a randomly generated binary integer matrix. The matrix map is responsible for the crossover process by determining the elements in the matrix $\mathbf{T}$ to be manipulated. The size of map is (Npop $\times$ Dim). The BTSO then performs a boundary check for the elements of the solution matrix $\mathbf{T}$ that violate the allowed limits of the search space after mutation and crossover. Finally, the global minimum is selected based on the objective to be minimized.

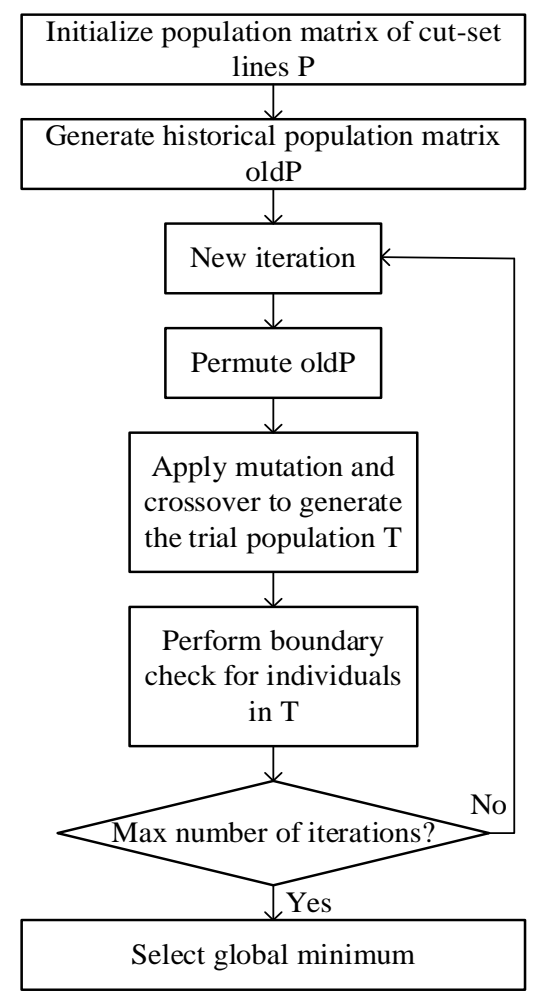

Fig. 2. Flowchart of the backtracking search optimization used for the design.

The BTSO has a simple structure that is fast, effective and can quickly adapt to different optimization problems. Unlike several evolutionary algorithms, the BTSO has a single control parameter, $F$, where the problem-solving process is not sensitive to the initial value of this parameter. This controlling parameter can produce small-amplitude numerical values required for a local search or large-amplitude numerical values that are necessary for a global search. The BTSO has not been adopted as a solution algorithm in the microgrid planning studies available in the literature.

\section{SimUlation RESUltS}

The selected test case is the PG\&E radial distribution system with 69 buses and one supply point as shown in Fig. 3.

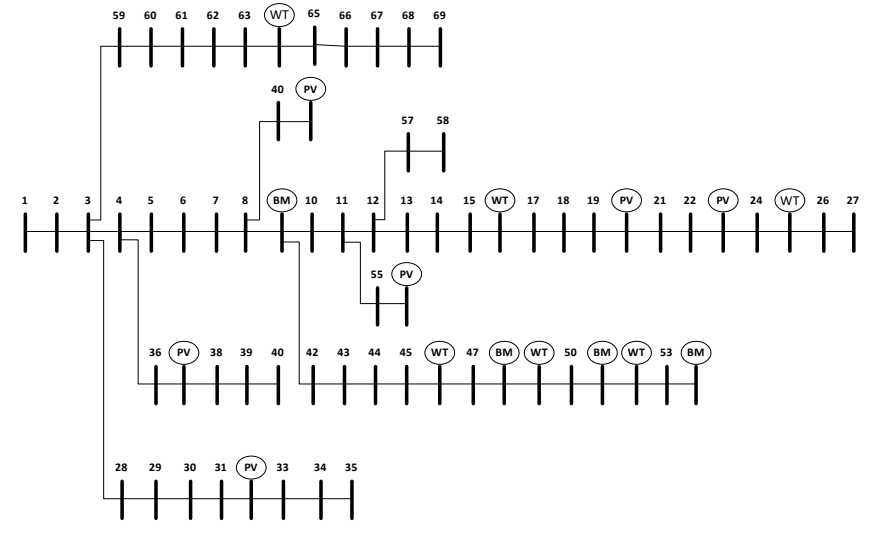

Fig. 3. System under study.

The system has a typical combination of $D G s$ as follows; 350 $\mathrm{kW}$ of $W T s, 175 \mathrm{~kW}$ of $P V s$, and $550 \mathrm{~kW}$ of $B M$ units. Details of the installed $D G s$ are given in Table I. The system loads are shown in Fig. 4.

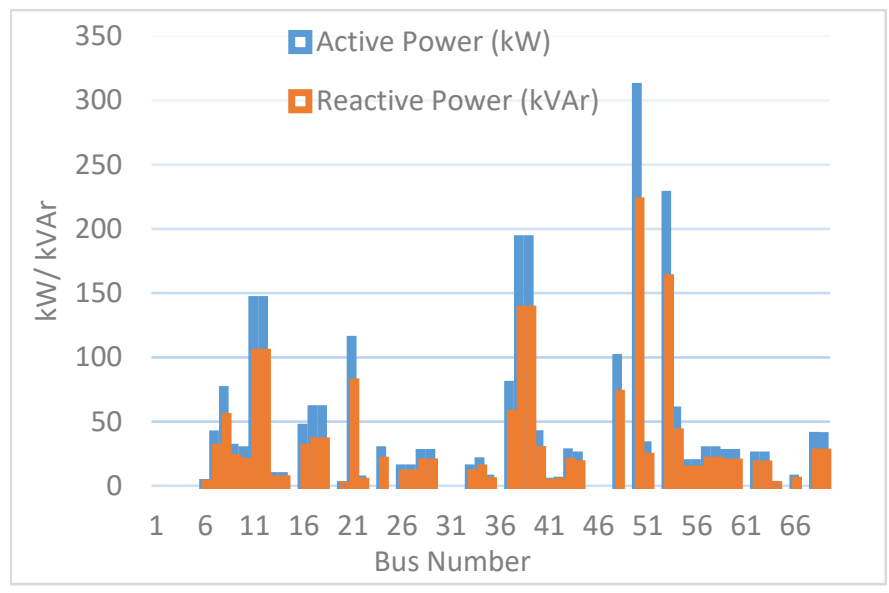

Fig. 4. Real and reactive power of system loads.

\begin{tabular}{ccc}
\multicolumn{3}{c}{ TABLE I } \\
\multicolumn{3}{c}{ INSTALLED DGS: CAPACITIES AND LOCATIONS } \\
\hline \hline DG & Locations/Buses & Capacities $(\mathrm{kW})$ \\
Units & $48,9,54,51$ & $125,50,200,175$ \\
\hline BM & $23,32,56,20,41$ & $25,50,25,25,25$ \\
PV & $16,46,52,64,25,49$ & $50,75,100,25,25,75$ \\
WT &
\end{tabular}

$D G s$ are more concentrated close to large loads, that are, buses $50,53,38$, and 39 . The $D G s$ and loads are represented using the probabilistic model elaborated in Section III. The algorithms adopted are the BTSO as the primary solution method, the shortest path method for sub-optimization problems, and the forward-backward sweep probabilistic load flow approach [41]. The system is then partitioned to determine the optimum microgrids for each objective. As explained in the previous sections, the optimal partitioning scenario for the first objective is the design with the lowest power imbalance in all zones. For the second objective, the optimal design scenario is the one with the highest probability for the successful operation of all zones when islanding occurs. 


\section{A. Application of the microgrid self-adequacy objective $\left(F_{1}\right)$}

The distribution system, shown in fig. 3 , is divided into multiple microgrids. Parameters in (7) are set as $(a=1, b=$ 0 ). The chosen values for the weights in (1) are $K_{1}=K_{2}=0.5$. The reason for this choice is that a high mismatch in the $Q$ power can have a bad impact on the bus voltages in the islands. From a mathematical perspective, the objective function will be minimized if the system is partitioned into only two microgrids, such that there is only one cut-set power line carrying the minimum power.

For more practical results, a constraint is added which limits the number of microgrids to five or more; that is, $N_{\text {microgrids }} \geq$ 5. This constraint is also valid to all simulations present in this paper. The optimum cut-set lines are shown in Table II. The cut-set line no. $y$ is the line ending with bus no. $(y+1)$; for example, the boundary line no. 62 is the line ending with bus no. 63. As shown in Table II, if it is required to partition the system into nine microgrids, that is, $N_{\text {microgrids }}=9$, the optimal eight cut-set lines that minimize the exchange of power between microgrids are $(3,8,10,13,20,28,46,62)$ and the corresponding value of the objective function $F_{1}$ is $264.42 \mathrm{pu}$. It is also evident that the value of $F_{1}$ increases as $N_{\text {microgrids }}$ increases. In [25], the $T S$ algorithm is applied to partition the PG\&E system into five, six, seven, eight, and nine microgrids. For comparison, the computed results from [25] are presented in Table III. A flowchart of the $T S$ algorithm is shown in Fig. 5 and a full description of the approach can be found in [25].

TABLE II

\begin{tabular}{|c|c|c|}
\hline $\mathrm{N}_{\text {microgrids }}$ & $\begin{array}{l}\text { Optimum virtual cut-set lines/ } \\
\text { virtual boundaries }\end{array}$ & $\mathrm{F}_{1}$ \\
\hline 5 & $19,28,46,62$ & 62.01 \\
\hline 6 & $13,20,28,46,62$ & 86.31 \\
\hline 7 & $10,13,20,28,46,62$ & 139.02 \\
\hline 8 & $8,10,13,20,28,46,62$ & 185.08 \\
\hline 9 & $3,8,10,13,20,28,46,62$ & 264.42 \\
\hline
\end{tabular}

TABLE III

OPTIMAL DESIGN USING TS [25] $-F_{1}$

\begin{tabular}{ccc}
\hline \hline $\mathrm{N}_{\text {microgrids }}$ & Optimum virtual cut-set lines & $\mathrm{F}_{1}$ \\
\hline $\mathbf{5}$ & $\mathbf{1 2 , \mathbf { 1 9 } , \mathbf { 2 8 , } \mathbf { 6 2 }}$ & $\mathbf{9 0 . 1 2}$ \\
6 & $9,12,19,28,62$ & 156.06 \\
7 & $9,12,19,28,47,62$ & 141.99 \\
8 & $8,9,12,19,28,47,62$ & 187.63 \\
9 & $3,8,9,12,19,28,47,62$ & 266.65 \\
\hline \hline
\end{tabular}

B. Application of the islanding success probability objective $\left(F_{2}\right)$

The system, shown in Fig. 3, is divided into a set of islands to maximize $F_{2}=I N D_{s y s}$ (same as minimizing $\left(1-F_{2}\right)$ ). The microgrid success indicator $F_{2}$ gives the probability of having successful islands taking into consideration the power requirements and the voltage limits of the microgrids. The parameters in (7) are set as $(a=0, b=1)$. Table IV shows the optimal switch locations, the corresponding values of success indicators $F_{2}$, as well as the corresponding values of $1-F_{2}$.

In Table IV, clustering the system into five islands, for example, has a success indicator $F_{2}$ equal to $59.42 \%$. The

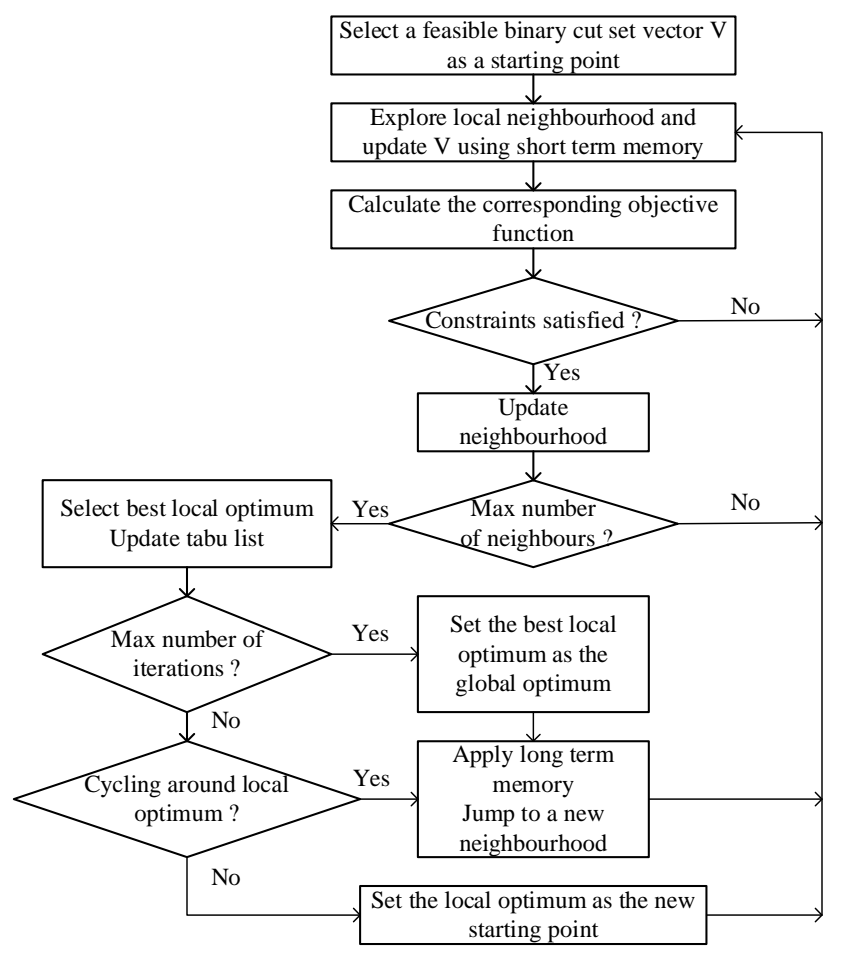

Fig. 5. Flowchart of the TS algorithm used for the microgrid design.

TABLE IV

OPTIMAL DESIGN USING BTSO $-F_{2}$

\begin{tabular}{cccc}
\hline \hline $\mathrm{N}_{\text {microgrids }}$ & $\begin{array}{c}\text { Optimum virtual cut- } \\
\text { set lines }\end{array}$ & $1-\mathrm{F}_{2}$ & $\begin{array}{c}\text { Microgrid success } \\
\text { indicator } \mathrm{F}_{2}\end{array}$ \\
\hline $\mathbf{5}$ & $\mathbf{1 0 , 1 3 , \mathbf { 2 0 , 6 2 }}$ & $\mathbf{0 . 4 0 5}$ & $\mathbf{5 9 . 4 2 \%}$ \\
6 & $10,13,20,28,62$ & 0.507 & $49.28 \%$ \\
7 & $3,10,13,20,28,62$ & 0.623 & $37.68 \%$ \\
8 & $4,10,13,20,28,41,62$ & 0.722 & $27.78 \%$ \\
9 & $3,6,10,13,20,28,41,62$ & 0.747 & $25.21 \%$ \\
\hline \hline
\end{tabular}

corresponding value of $\left(1-F_{2}\right)$ is $0.405 \mathrm{pu}$. The value of $F_{2}$ decreases as the number of microgrids increases.

Next, the robustness of using a single objective for partitioning of distribution systems is examined. The corresponding values of $F_{2}$ and $\left(1-F_{2}\right)$ are calculated for the optimal design in Table III. For further explanation, if it is required to partition the system into five microgrids where (12, $19,28,62$ ) are the cut-set lines, it is worth knowing the success probability of the designed microgrids if islanding is to occur. The corresponding values of $F_{2}$ and $\left(1-F_{2}\right)$ are calculated using (6). The results are, presented in Table $\mathrm{V}$ (calculated values in bold).

TABLE V

OPTIMAL DESIGN USING TS [25] - CALCULATED $F_{2}$ and $1-F_{2}$

\begin{tabular}{ccccc}
\hline \hline \multirow{2}{*}{$\mathrm{N}_{\text {microgrids }}$} & $\begin{array}{c}\text { Optimum virtual } \\
\text { cut-set lines }\end{array}$ & $\begin{array}{c}\mathrm{F}_{1} \\
(\mathrm{pu})\end{array}$ & $\begin{array}{c}\mathbf{1}-\mathbf{F}_{2} \\
\mathbf{( p u )}\end{array}$ & $\begin{array}{c}\text { Microgrids } \\
\text { success } \\
\text { indicator } \\
\mathbf{F}_{2}\end{array}$ \\
\hline 5 & $12,19,28,62$ & 90.12 & $\mathbf{0 . 4 2 0}$ & $\mathbf{5 7 . 9 7 \%}$ \\
6 & $9,12,19,28,62$ & 156.06 & $\mathbf{0 . 5 2 1}$ & $\mathbf{4 7 . 8 3 \%}$ \\
7 & $9,12,19,28,47,62$ & 141.99 & $\mathbf{0 . 8 9 8}$ & $\mathbf{1 0 . 1 4 \%}$ \\
8 & $8,9,12,19,28,47,62$ & 187.63 & $\mathbf{0 . 8 0 1}$ & $\mathbf{1 9 . 8 3 \%}$ \\
9 & $3,8,9,12,19,28,47,62$ & 266.65 & $\mathbf{0 . 8 0 1}$ & $\mathbf{1 9 . 8 3 \%}$ \\
\hline \hline
\end{tabular}

Similarly, the values of $F_{1}$ are computed for the optimal design in Table IV. For further elaboration, if it is required to partition the system into five microgrids where $(10,13,20,62)$ 
are the cut-set lines. It is worth inspecting the self-adequacy of these microgrids during the grid-connected mode of operation. The calculated values are shown in Table VI (calculated values in bold).

TABLE VI

OPTIMAL DESIGN USING BTSO - calculated $F_{1}$

\begin{tabular}{ccccc}
\hline \hline $\mathrm{N}_{\text {microgrids }}$ & $\begin{array}{c}\text { Optimum virtual } \\
\text { cut-set lines }\end{array}$ & $\begin{array}{c}1-\mathrm{F}_{2} \\
(\mathrm{pu})\end{array}$ & $\begin{array}{c}\text { Microgrid } \\
\text { success } \\
\text { indicator } \\
\mathrm{F}_{2}\end{array}$ & $\begin{array}{c}\mathbf{F}_{\mathbf{1}} \\
\mathbf{( p u )}\end{array}$ \\
\hline 5 & $12,19,28,62$ & 0.405 & $59.42 \%$ & $\mathbf{1 8 3 . 6 2}$ \\
6 & $9,12,19,28,62$ & 0.507 & $49.28 \%$ & $\mathbf{1 5 2 . 5 1}$ \\
7 & $9,12,19,28,47,62$ & 0.623 & $37.68 \%$ & $\mathbf{2 6 3 . 7 2}$ \\
8 & $8,9,12,19,28,47,62$ & 0.722 & $27.78 \%$ & $\mathbf{1 9 8 . 3 9}$ \\
9 & $3,8,9,12,19,28,47,62$ & 0.747 & $25.21 \%$ & $\mathbf{2 7 5 . 7 2}$ \\
\hline \hline
\end{tabular}

As shown in Table $\mathrm{V}$, for $N_{\text {microgrids }}=5$, using $F_{1}$ as a single objective to solve the design problem results in the cutset lines $(12,19,28,62)$ and power imbalance equal to 90.12 $\mathrm{pu}$. The calculated corresponding microgrid success indicator for the cut-set line $(12,19,28,62)$ is $57.97 \%$. While, in Table VI, for $N_{\text {microgrids }}=5$, using $F_{2}$ as a single objective to solve the design problem results in the cut-set lines $(10,13,20,62)$ and a microgrid success indicator equal to $59.42 \%$. The calculated corresponding power imbalance $F_{1}$ is equal to 183.62 pu. By comparing all the values in Tables V and VI, in the same manner, it can be concluded that considering only the objective $F_{2}$ for the design of microgrids achieves higher success islanding success probability but more power imbalance than when considering only the objective $F_{1}$. Likewise, considering only the objective $F_{1}$ for the design achieves less power imbalance than when considering the objective $F_{2}$ but lower islanding success probability. That is why $F_{3}$ is proposed.

\section{Application of the combined objective $\left(F_{3}\right)$.}

In this subsection, the objective function $F_{3}$ is considered, which combines both objectives $F_{1}$ and $F_{2}$ together using a weighted summation. As mentioned in Section IV, the parameters $a$ and $b$ in (7) can be changed based on the importance of each objective for the considered system. The values of the parameters are set as $a=b=0.5$ to study the effect of combining both objectives equally. The 69-bus system under study is partitioned into five, six, seven, eight, and nine microgrids using the BTSO. The optimum cut-set lines, as well as the values of $F_{3}, F_{2}$, and $F_{1}$, are given in Table VII.

Fig. 6 illustrates the electrical boundaries of the designed TABLE VII OPTIMAL DESIGN USING BTSO $-F_{3}, F_{2}$, and $F_{1}$

\begin{tabular}{cccccc}
\hline \hline $\begin{array}{c}\mathrm{N}_{\text {micro- }} \\
\text { grids }\end{array}$ & $\begin{array}{c}\text { Optimum virtual } \\
\text { cut-set lines }\end{array}$ & $\begin{array}{c}\mathrm{F}_{3} \\
(\mathrm{pu})\end{array}$ & $\begin{array}{c}\mathrm{F}_{1} \\
(\mathrm{pu})\end{array}$ & $\begin{array}{c}1-\mathrm{F}_{2} \\
(\mathrm{pu})\end{array}$ & $\begin{array}{c}\text { Microgrid } \\
\text { success } \\
\text { indicator } \\
\mathrm{F}_{2}\end{array}$ \\
\hline 5 & $13,20,28,62$ & 65.28 & 90.00 & 0.4058 & $59.42 \%$ \\
6 & $\begin{array}{c}10,13,20,28,62 \\
7\end{array}$ & 101.61 & 152.51 & 0.5072 & $49.28 \%$ \\
7 & $\begin{array}{c}10,13,20,28,41, \\
62\end{array}$ & 110.11 & 139.06 & 0.8116 & $18.84 \%$ \\
8 & $\begin{array}{c}\text { 8, 10, 13, 20, 28, } \\
46,62\end{array}$ & 131.90 & 185.08 & 0.7873 & $21.27 \%$ \\
$\mathbf{9}$ & $\begin{array}{c}\mathbf{3 , 8 , 1 0 , 1 3 , 2 0 ,} \\
\mathbf{2 8 , 4 6 , 6 2}\end{array}$ & $\mathbf{1 7 1 . 5 7}$ & $\mathbf{2 6 4 . 4 2}$ & $\mathbf{0 . 7 8 7 3}$ & $\mathbf{2 1 . 2 7 \%}$ \\
\hline
\end{tabular}

islands when the system is partitioned into nine microgrids using the objective function $F_{3}$. From the self-adequacy perspective, all the values of $F_{1}$ obtained by the BTSO in Table VII are less than their corresponding values obtained by the $T S$ in Table V. Also, all the success indicator values $F_{2}$ in Table VII obtained by the BTSO are higher than their corresponding values in Table $\mathrm{V}$ obtained by the $T S$. Thus, we concluded that using the BTSO to solve the microgrid design problem under study can be more efficient than using $T S$.

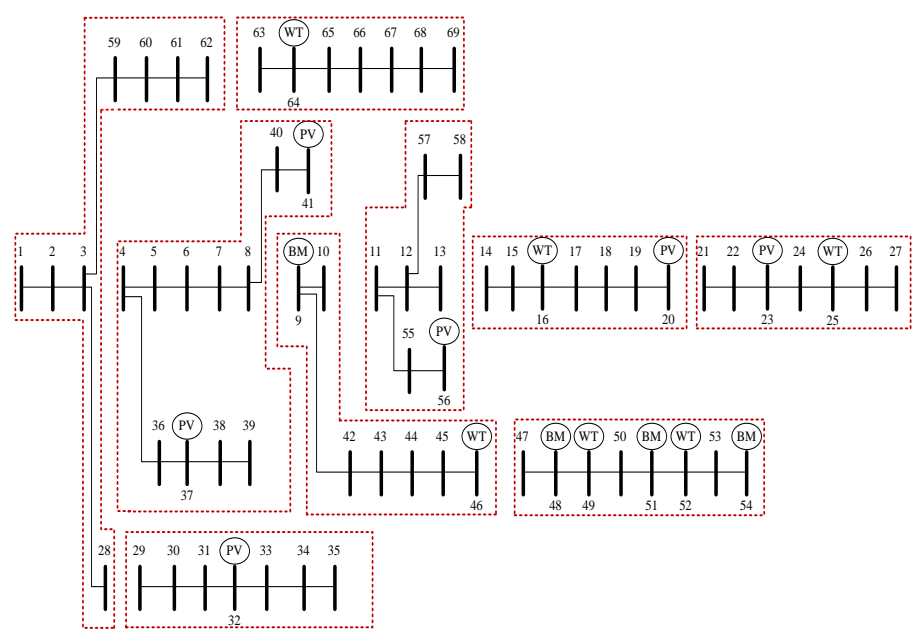

Fig. 6. Electric boundaries of the designed microgrids for $N_{\text {microgrids }}=9$

\section{Effect of variation of optimization coefficients}

In this subsection, the impact of the change of the optimization coefficients $a$ and $b$ in (7) on the final constructed microgrids is studied. The 69-bus system is clustered into five microgrids where the coefficients are given different values in the interval $[0,1]$ such that the condition $a+b=1$ is satisfied in each case. Results are given in Table VIII.

TABLE VIII

EFFECT OF OPTIMIZATION COEFFICIENTS ON THE OPTIMAL DESIGN $\left(N_{\text {microgrids }}=5\right)$

\begin{tabular}{ccccccc}
\hline \hline $\mathrm{a}$ & $b$ & $\begin{array}{c}\text { Optimum virtual cut- } \\
\text { set lines }\end{array}$ & $\begin{array}{c}\mathrm{F}_{3} \\
(\mathrm{pu})\end{array}$ & $\begin{array}{c}\mathrm{F}_{1} \\
(\mathrm{pu})\end{array}$ & $\begin{array}{c}1-\mathrm{F}_{2} \\
(\mathrm{pu})\end{array}$ & $\begin{array}{c}\text { Microgrid } \\
\text { success } \\
\text { indicator } \\
\mathrm{F}_{2}\end{array}$ \\
\hline 0.1 & 0.1 & $13,20,28,62$ & 45.52 & 90.00 & 0.4058 & $59.42 \%$ \\
0.3 & 0.3 & $13,20,28,62$ & 55.40 & 90.00 & 0.4058 & $59.42 \%$ \\
0.6 & 0.6 & $19,28,41,62$ & 69.56 & 62.08 & 0.8078 & $19.22 \%$ \\
0.7 & 0.7 & $19,28,41,62$ & 67.69 & 62.08 & 0.8078 & $19.22 \%$
\end{tabular}

As the parameter $a$ increases and the parameter $b$ decreases, the value of $F_{1}$ decreases and the value of the $F_{2}$ increases. The reason is that the overall objective function $F_{3}$ is now more directed towards minimizing the power imbalance during the design. The optimum boundary lines vary with the variation of coefficients. Thus, in order to have more practical and costefficient results, it is recommended that the optimization coefficients to be chosen based on a cost-benefit analysis. 


\section{E. Comparison with other microgrid design objectives}

Several objectives have been used in the previous literature for the optimal design of microgrids. Some modifications are introduced for the system under study for comparison. The updated loads and the new distribution of $D G s$ are presented in Fig. 7 and Table IX respectively.

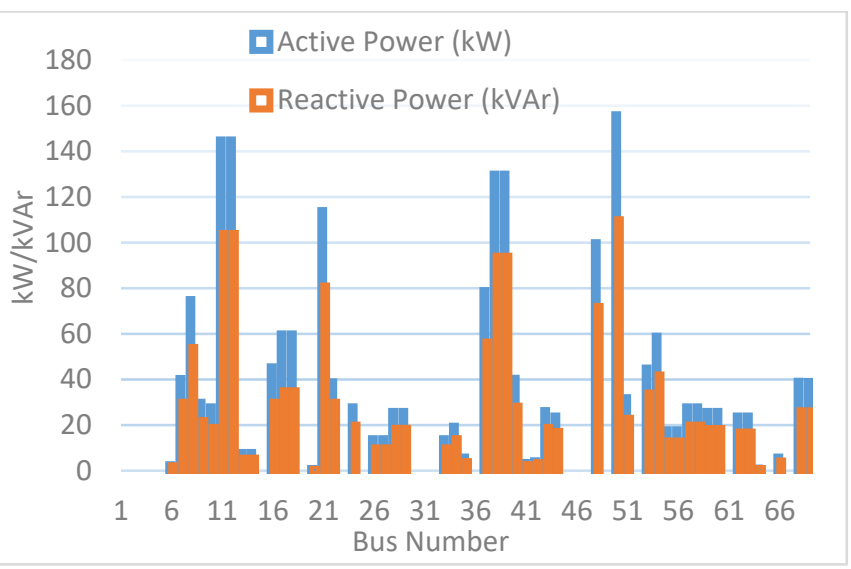

Fig. 7. Modified system loads

TABLE IX

MODIFIED DISTRIBUTION OF DGS: CAPACITIES AND LOCATIONS

\begin{tabular}{cll}
\hline \hline DG & \multicolumn{1}{c}{ Locations/ Buses } & \multicolumn{1}{c}{ Capacities $(\mathrm{kW})$} \\
Units & \multicolumn{1}{c}{ (15, 21, 27, 33, 38, 42, 45, } & $25,50,25,50,75,50,50,50$, \\
\hline$B M$ & $6,15,75,75$ \\
& $54,57,68$ & $25,25,25,25,25,25$ \\
$P V$ & $30,36,50,56,58,62$ & $50,25,25,50,50,50$ \\
$W T$ & $13,16,19,35,43,52$ & \\
\hline \hline
\end{tabular}

$25 \mathrm{~kW}$ of $E S U s$ are added to the system to mitigate the effect of intermittent $D G s$ by storing the spilled energy of renewable resources. Furthermore, $25 \mathrm{kVAr}$ of $R S s$ are also introduced to the system to supply reactive power, improve bus voltages, reduce losses and increase the overall capacity of the system [46]. The locations and number of installed ESUs and $R S s$ at each bus are given in Table $\mathrm{X}$. The power of $E S U S\left( \pm P_{E S U}\right)$ and $R S S\left(Q_{R S}\right)$ are used to modify the load flow equations. $P_{E S U}$ is added to the generated active power during the discharging period and to the active load demand during the charging period. $Q_{R S}$ is added to the generated reactive power with specific rated capacity.

The system is then optimally partitioned into sets of microgrids using the BTSO. A comparison with other objectives that are present in the previous literature is shown in Table XI. In [27], the TS is employed to partition the modified test system into control zones. The considered objectives are the communication network's properties and costs as well as the power imbalance in the microgrids. The design in [42] takes into account the reliability and supply-security objectives.

It is clear that the optimal design varies with the considered objective. The optimum boundaries are also sensitive to the penetration level of distributed resources, ESUs, and RSs.

Fig. 8 shows the optimal infrastructure when partitioning the modified system into six microgrids with maximized selfadequacy and islanding success probability. In this case, the virtual cut set lines (indicated in bold in Table XI) are $(13,20$,
TABLE X

INSTALLED DGS: CAPACITIES AND LOCATIONS

\begin{tabular}{ccc}
\hline \hline \multirow{2}{*}{ Units } & Locations & No. of units installed \\
\hline \multirow{2}{*}{ ESUs } & $7,18,30,36,37,39,35,38,56,59$, & $1,1,1,2,1,2,1,1,1,3$, \\
& 61,63 & 2,2 \\
\multirow{2}{*}{ RSs } & $7,11,15,19,23,31,33,35,39,43$, & $1,2,1,1,1,1,1,1,1,1$, \\
& $47,51,62,67$ & $2,1,1,1$ \\
& & \\
\hline
\end{tabular}

TABLE XI

DESIGN RESULTS OF THE MODIFIED TEST SYSTEM USING DIFFERENT OBJECTIVES

\begin{tabular}{|c|c|c|}
\hline Objective & $\mathrm{N}_{\text {micro- }}$ & $\begin{array}{l}\text { Optimum virtual cut. } \\
\text { set lines }\end{array}$ \\
\hline $\begin{array}{l}\text { Self-adequacy and islanding success } \\
\text { probability }\end{array}$ & 5 & $18,27,41,59$ \\
\hline Power imbalance [27] & 5 & $6,15,48,59$ \\
\hline Communication and control [27] & 5 & $8,18,9,61$ \\
\hline Reliability [42] & 6 & $8,18,28,42,60$ \\
\hline $\begin{array}{l}\text { Self-adequacy and islanding success } \\
\text { probability }\end{array}$ & 6 & $13,20,27,41,59$ \\
\hline Supply Security [42] & 6 & $3,6,10,15,48$ \\
\hline
\end{tabular}

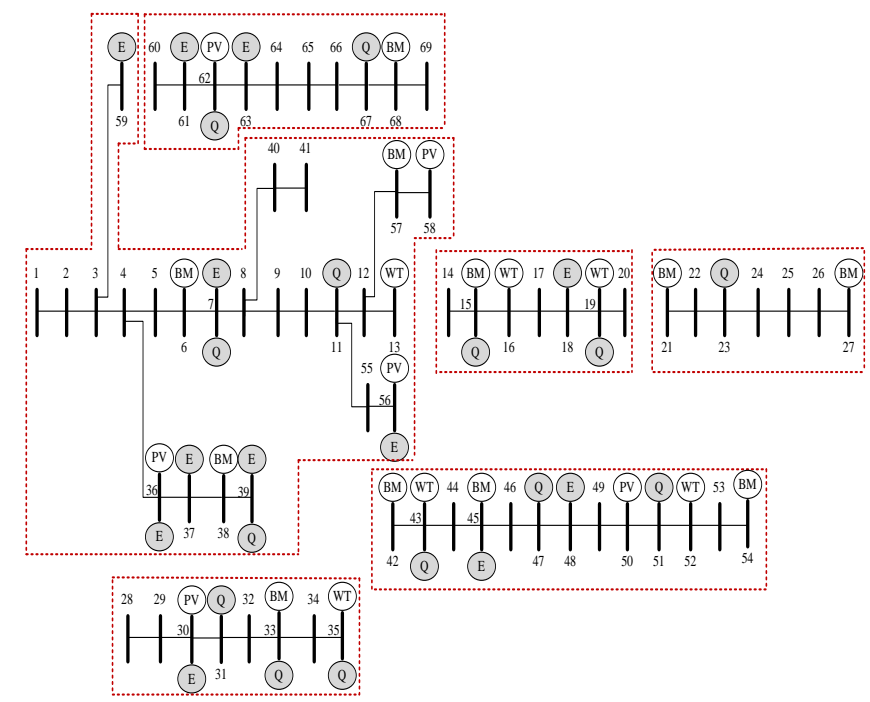

Fig. 8. Optimal infrastructure of the modified test system $\left(\mathrm{N}_{\text {microgrids }}=6\right)$

$7,41,59)$. The corresponding value of the combined objective function $F_{3}$ is $57.05 \mathrm{pu}$ with microgrids islanding success indicator $F_{2}$ of $94.2 \%$ and power mismatch $F_{1}$ of $51.32 \mathrm{pu}$.

\section{DISCUSSIONS}

In the previous section, clustering of the PG\&E 69-bus distribution system into networked microgrids is scrutinized based on different technical criteria.

First, the self-sufficiency of microgrids is maximized such that each microgrid operates with the least amount of energy exchange with other microgrids during the grid-connected mode. If islanding is to occur, a minimum number of load shedding action will then be required. Comparing the results in Table II and Table III, the BTSO obtains improved results than the $T S$ such that the imbalance objective function $F_{1}$ is reduced by 31.1, 44.6, 2, 1.3, and $0.8 \%$ when clustering the system into five, six, seven, eight, and nine microgrids respectively.

After that, the system is partitioned to maximize the probability of successful operation of microgrids during 
islanding. The results indicate that the optimum cut-set lines vary with the considered objective. When comparing the $T S$ results in Table $\mathrm{V}$ with the simulation results obtained in Table IV, the success indicator is improved by $1.45,1.4,27.5,7.9$, and $5.38 \%$ when clustering the system into five, six, seven, eight, and nine microgrids respectively. Also, it can be concluded that using the success indicator as a single objective maximizes the probability of a successful islanding operation on account of the self-adequacy of the microgrids and that is why a combined objective function is proposed. Both the self-adequacy and the islanding success objectives are combined in a single objective function as a normalized weighted summation. The new objective function ensures that the microgrid's different modes of operation, that is, grid-connected or standalone, are considered in the design process. The optimal design is the one with the smallest power imbalance and highest probability of successful islanding operation of all zones. The optimum cutset lines are different from those obtained by using each objective individually. Both the self-adequacy and the microgrid success indicators are improved compared to the TS results in Table V. In Table VII, the power imbalance is improved by $0.1,2.2,2,1.3$, and $0.8 \%$ and the success indicator is improved by $1.4,1.4,8.7,1.4$, and $1.4 \%$ when clustering the system into five, six, seven, eight, and nine microgrids respectively. Sensitivity analysis reveals that the optimum cutset lines are dependent on the system's requirements.

In all simulations, the BTSO managed to find a better global optimal solution than the TS. The local search procedures that the TS is based on can get stuck in a poor neighborhood search area. TS utilizes a tabu list as well as other several forms of memories for intensification and diversification, e.g., shortterm and long-term memories. However, these types of memories can overlap and may require the determination of many parameters where the number of iterations could be considerably large. As a result, TS may fail to find the optimal global solution depending on the parameters settings.

Finally, the distribution of $P V, W T, B M$ units, and loads along the studied system buses is modified. ESUS and $R S s$ are added to different buses. The BTSO is then applied to partition the system optimally. Comparison in Table XI with other objectives available in the previous literature reveals that the constructed microgrids are different based on the goal of the design. The optimal interconnection of microgrids is also sensitive to the increase of load demand and the penetration level of DGs.

\section{CONCLUSIONS AND FUTURE WORKS}

In this paper, we present a systematic planning framework to develop conventional distribution networks using interconnected microgrids. Various concerns that affect the optimal interconnection of microgrids are addressed such as active and reactive power imbalance, microgrids islanding success, intermittency of generation, load variability and employment of ESUs and RSs. The BTSO is utilized to determine the optimal electrical boundaries of the constructed microgrids. The proposed planning approach can help utility planners to upgrade conventional distribution networks using the virtual microgrids concept. This work can be considered as one of the solutions to the restrictions imposed by the infrastructure of present grids on the adoption of reliable and self-healing smart grids. Future extension of this work can include the application of the proposed approach to large distribution systems, and the inclusion of other technical concerns such as the expected load growth, the future increase in the penetration level of renewable $D G s$, and flexibility of microgrids' boundaries under contingencies.

\section{REFERENCES}

[1] D. Quiggin, S. Cornell, M. Tierney, and R. Buswell, “A simulation and optimisation study: Towards a decentralised microgrid, using real world fluctuation data," Energy, vol. 41, no. 1, pp. 549-559, May 2012.

[2] H. Jiayi, J. Chuanwen, and X. Rong, "A review on distributed energy resources and Microgrid,” Renew. Sust. Energy. Rev., vol. 12, no. 9, pp. 2472-2483, Dec. 2008.

[3] X. Tan, Q. Li, and H. Wang, "Advances and trends of energy storage technology in microgrid,” Int. J. Elec. Power, vol 44, no. 1, pp. 179-191, Jan. 2013.

[4] W. Su, and J. Wang, "Energy management systems in microgrid operations,” Electr. J., vol. 25, no. 8, pp. 45-60, Oct. 2012.

[5] D. E. Olivares et al., "Trends in microgrid control," IEEE Trans. Smart Grid, vol. 5, no. 4, pp. 1905-1919, Jul. 2014.

[6] S. Wang et al., "New metrics for assessing the reliability and economics of microgrids in distribution system," IEEE Trans. Power Syst., vol. 28, no. 3, pp. 2852-2861, Aug. 2013.

[7] A. Mohsenzadeh, C. Pang, and M. Haghifam, "Determining optimal forming of flexible microgrids in the presence of demand response in smart distribution systems," IEEE Syst. J., to be published.

[8] F. Farzan et al., "Microgrids for fun and profit: The economics of installation investments and operations," IEEE Power Energy Magazine, vol. 11, no. 4, pp. 52-58, Jul.-Aug. 2013.

[9] S. S. Reddy, J. Y. Park, and C. M. Jung, “Optimal operation of microgrid using hybrid differential evolution and harmony search algorithm,” Front. Energy, vol. 10, no. 3, pp. 355-362, Sept. 2016.

[10] M. H. Oboudi, R. Hooshmand, and A. Karamad, "A feasible method for controlled intentional islanding in microgrids based on PSO algorithm," Swarm Evol. Comput., vol. 35, pp. 14-25, Aug. 2017.

[11] IEEE standard for interconnecting distributed resources with electric power systems, Jul. 28, 2003, IEEE Std 1547-2003.

[12] M. Golari, N. Fan, and J. Wang, "Two-stage stochastic optimal islanding operations under severe multiple contingencies in power grids," Electr. Power Syst. Res., vol. 114, pp. 68-77, Sep. 2014.

[13] J. Zhao, H. Niu, and X. Zhang, "Island partition of distribution network with microgrid based on the energy at risk,” IET Gener. Transm. Dis., vol. 11, no. 4, pp. 830-837, Sep. 2017.

[14] G. Xu, S. Wu, and Y. Tan, "Island partition of distribution system with distributed generators considering protection of vulnerable nodes,” Appl. Sci., vol. 7, no. 10, pp. 1057, Oct. 2017.

[15] IEEE Guide for design, operation, and integration of distributed resource island systems with electric power systems, Jul. 20, 2011, IEEE Std 1547.4-2011.

[16] R. H. Lasseter, "Smart distribution: coupled microgrids," Proc. IEEE, vol. 99, no.6, pp. 1074-1082, Jun. 2011.

[17] A. Khodaei, S. Bahramirad, and M. Shahidehpour, "Microgrid planning under uncertainty," IEEE Tran. Power Syst., vol.30, no.5, pp. 2417-2425, Sep. 2015.

[18] F. S. Gazijahani, and J. Salehi, "Robust design of microgrids with reconfigurable topology under severe uncertainty," IEEE Trans. Sustainable Energy, vol. 9, no.2, pp. 559-569. Apr. 2018. 
[19] A. Narayan, and K. Ponnambakam, "Risk-averse stochastic programming approach for microgrid planning under uncertainty,” Renewable Energy, vol. 101, pp. 399-408, Feb. 2017.

[20] F. S. Gazijahani, and J. Salehi, "Reliability constrained twostage optimization of multiple renewable based microgrids incorporating critical energy peak pricing demand response program using robust optimization approach,” Energy, vol. 161, pp. 999-1015, Oct. 2018.

[21] H. Lotfi, and A. Khodaei, "AC versus DC microgrid planning,” IEEE Trans. Smart Grid, vol. 8, no.1, pp. 296304, Jan. 2017.

[22] F. S. Gazijahani, and J. Salehi, “Optimal bilevel model for stochastic risk-based planning of microgrids under uncertainty,” IEEE Trans Ind. Informat., vol. 14, no.7, pp. 3054-3064, Jul. 2018.

[23] F. S. Gazijahani, and J. Salehi, "Stochastic multi-objective framework for optimal dynamic planning of interconnected microgrids,” IET Renew. Power Gener., vol. 11, no. 14, pp. 1749-1759, Dec. 2017.

[24] M. Barani, J. Aghaei, M. Akbari, T. Niknam, H. Farahmand, and M. Korpas, "Optimal partitioning of smart distribution systems into supply-sufficient microgrids,” IEEE Trans. Smart Grid, Accepted for publication, 10.1109/TSG.2018.2803215.

[25] S. A. Arefifar, Y. A. I. Mohamed, and T. H. M. El-Fouly, "Supply-adequacy-based optimal construction of microgrids in smart distribution systems,” IEEE Trans. Smart Grid, vol. 3, no. 3, pp. 1491-1502, Sept. 2012.

[26] S. A. Arefifar, and Y. A. I. Mohamed, "DG mix, reactive sources and energy storage units for optimizing microgrid reliability and supply security,” IEEE Trans. Smart Grid, vol.5, no. 4, pp. 1835-1844, Jul. 2014.

[27] S. A. Arefifar, Y. A. I. Mohamed, and T. H. M. El-Fouly, "Optimized multiple microgrid-based clustering of active distribution systems considering communication and control requirements,” IEEE Trans. Ind. Electron., vol. 62, no. 2, pp. 711-723, Feb. 2015.

[28] H. Haddadian, and R. Noroozian, "Multi-microgrids approach for design and operation of future distribution networks based on novel technical indices,” Applied Energy, vol. 185, pp. 650-663, Jan. 2017.

[29] P. Civicioglu, "Backtracking search optimization algorithm for numerical optimization problems,” Appl. Math. Comput., vol. 219, no. 15, pp. 8121-8144, Apr. 2013.

[30] M. Shahraeini, M. H. Javidi, and M. S. Ghazizadeh, "Comparison between communication infrastructures of centralized and decentralized wide area measurement systems,” IEEE Trans. Smart Grid, vol. 2, no. 1, pp. 206211, Mar. 2011.

[31] S. A. Arefifar, Y. A. I. Mohamed, and T. El-Fouly, "Comprehensive operational planning framework for selfhealing control actions in smart distribution grids," IEEE Trans. Power Syst., vol. 28, no.4, pp. 4192-4200, Nov. 2013.

[32] S. S. Reddy, P. R. Bijwe, and A. R. Abhyankar, "optimum day-ahead clearing of energy and reserve markets with wind power generation using anticipated real-time adjustment costs,” Int. J. Elec. Power, vol. 71, pp. 242-253, Oct. 2015.

[33] S. S. Reddy, V. Sandeep, and C.-M. Jung, "Review of stochastic optimization methods for smart grid," Front. Energy, vol. 11, no.2, pp. 197-209, June 2017.

[34] S. S. Reddy, P. R. Bijwe, and A. R. Abhyankar, "Real time economic dispatch considering renewable energy resources," Renewable Energy, vol.83, pp. 1215-1226, Nov. 2015.

[35] S. S. Reddy, and J. A. Momoh, "Realistic and transparent optimum scheduling strategy for hybrid power system," IEEE Trans. Smart Grid, vol. 6, no.6, pp. 3114-3125, Nov. 2015.

[36] S. S. Reddy, P. R. Bijwe, and A. R. Abhyankar, "Real-time economic dispatch considering renewable power generation variability and uncertainty over scheduling period," IEEE Syst. J., vol. 9, no. 4, pp. 1440-1451, Dec. 2015.

[37] J. M. S. Pinheiro, C. R. R. Dornellas, M. Th. Schilling, A. C. G. Melo and J. C. O. Mello, "Probing the new IEEE reliability test system (RTS-96): HL-II assessment,” IEEE Trans. Power Syst., vol. 13, no. 1, pp. 171-176, Feb. 1998.

[38] X. Xu, F. Xue, S. Lu, H. Zhu, L. Jiang and B. Han, "Structural and hierarchical partitioning of virtual microgrids in power distribution network," IEEE Syst. J., to be published.

[39] Y. M. Atwa, E. F. El-Saadany, M. M. A. Salama, R. Seethapathy, M. Assam, and S. Conti, “Adequacy evaluation of distribution system including wind/solar DG during different modes of operation,” IEEE Trans. Power Syst., vol. 26, no.4, pp. 1945-1952, Nov. 2011.

[40] V. Cherkassy Boris, V. Goldberg Andrew, and R. Tomasz, "Shortest paths algorithms: theory and experimental evaluation,” Math. Program Ser. A, vol. 73, no. 2, pp. 129174, 1996.

[41] D. Shirmohammadi, H.W. Hong, A. Semlyen, and G. X. Luo, "A compensation-based power flow method for weakly meshed distribution and transmission networks," IEEE Trans. Power Syst., vol. 3, no. 2, pp. 753-762, May 1988.

[42] S. A. Arefifar, Y. A. I. Mohamed, and T. H. M. El-Fouly, "Optimum microgrid design for enhancing reliability and supply-security,” IEEE Trans. Smart Grid, vol. 4, no. 3, pp. 1567-1575, Sep. 2013.

[43] A. Pouryekta, V. K. Ramachandaramurthy, N. Mithulananthan, and A. Arulampalam, "Islanding detection and enhancement of microgrid performance,” IEEE Syst. J., to be published.

[44] R. A. Osama, A. Y. Abdelaziz, R. Swief, M. Ezzat, and A. F. Zobaa, "A probabilistic approach for maximizing the islanding success of microgrids," in Proc. MEPCON, Cairo, Egypt, 2017, pp. 392-396.

[45] S. Reddy, "Optimal Scheduling of thermal-wind-solar power system with storage,” Renewable Energy, vol. 101, pp. 1357-1368, Feb. 2017.

[46] S. S. Reddy, “Optimal power flow with renewable energy resources including storage,” Electr. Eng., vol. 99, no. 2, pp. 685-695, June 2017. 